\title{
SLC Solute Carrier Transporters and Neurodegenerative Disorders: Drawing Attention to Cationic Amino Acid Transporters 1 and 2
}

\author{
Hans-Gert Bernstein ${ }^{1}$, Gerburg Keilhoff ${ }^{2}$, Henrik Dobrowolny ${ }^{1}$, Johann Steiner ${ }^{1}$ \\ ${ }^{1}$ Department of Psychiatry, ${ }^{2}$ Institute of Biochemistry and Cell Biology, Faculty of Medicine, Otto-von-Guericke University, Magdeburg, Germany
}

\section{TO THE EDITOR}

We read with great interest the recently published review article by Aykaç and Şehirli [1] about the implication of several solute carrier (SLC) transporters for neurodegenerative disorders and wish to comment on selected aspects of this important topic. Drs. Aykaç and Şehirli succeeded in providing a good overview about this sprawling area of knowledge, whereby some SLC transporters subfamilies are discussed in greater detail, while others are less well elaborated. Unfortunately, SLC7 gene family transporters belong to the latter group. With regard to these transporter proteins the authors write: "Many subtypes of the SLC7 gene family are expressed in the brain. Particularly, they play an important role in diseases like schizophrenia and Parkinson's disease (PD) by influencing the distribution of the amine or amino acid structured neurotransmitter precursor known as L-DOPA". The paper contains a table, which aims to summarize information about the distribution of SLC proteins in the CNS and the disorders in which they play a role. Consulting this table one easily can see that of the known four (and perhaps, even five) members of cationic amino acid transporter proteins (CATs, which constitute a SLC7A subfamily) only two are listed as being expressed in the brain: CAT3 and CAT4. The question arises: What about the high affinity transporter CAT1 (SLC7A1) and the low affinity transporter CAT2 (SLC7A2)? Since both transporters are not only neglectfully treated in this overview, but also in some other recent publications on cerebral SLC trans-

Received: May 4, 2020 / Accepted: May 7, 2020

Address for correspondence: Hans-Gert Bernstein

Department of Psychiatry, Faculty of Medicine, Otto-von-Guericke

University, Leipziger Straße 44, Magdeburg 39120, Germany

E-mail: Hans-Gert.Bernstein@med.ovgu.de

ORCID: https://orcid.org/0000-0002-2594-1689 porters [2-5], we thought it's time to draw some attention to these two transporter proteins. Two requirements must be met before a transporter protein might be included into Aykaç's and Şehirli's "candidate list". The first question to be answered is: Are CAT1 and CAT2 expressed in the brain? The answer is clearly yes. Both CAT1 and CAT2 are widely expressed in rat and human brain. They are predominantly localized in neurons, but are also found in numerous astrocytes, oligodendrocytes, choroid plexus epithelial cells, and small blood vessels [6-9]. The second (and even more important) question is: Is there any evidence for an involvement of either CAT1 or CAT2 in neurodegeneration? Since Aykaç and Şehirli understand neurodegenerative disorders in a broad sense, the answer would also be yes for CAT1. A few years ago some of us could show that in postmortem brains of individuals with mood disorder (major depression and bipolar disorder) there is a significant decrease in the numerical density of human CAT1 expressing neurons in the CA2 region of the hippocampus. The functional importance of this finding is yet not fully clear, but has possibly to do with the downregulation of the endogenous antidepressant agmatine in depression [10, 11]. Concerning CAT2 (which appears in two isoforms, CAT2a and CAT2b) it can be stated that the transporter may play a role in the pathophysiology of a "classical" neurodegenerative disorder, Alzheimer's disease (AD). Colton and co-workers have reported that CAT2 mRNA is significantly upregulated in macrophages in $\mathrm{AD}$ and in a mouse model of the disease [12]. These immune cells are prominently involved in neuro-inflammatory processes, which are a core event in $\mathrm{AD}$ pathology. From the data compilation in Aykaç's and Şehirli's paper it is obvious that a vast majority of the SLC transporters (including the two cationic transporters listed, CAT3 and CAT4) do not play a known role in neurodegenerative

(ㄷ) This is an Open-Access article distributed under the terms of the Creative Commons Attribution Non-Commercial License (http://creativecommons.org/licenses/by-nc/4.0) which permits unrestricted non-commercial use, distribution, and reproduction in any medium, provided the original work is properly cited. 
disorders. This is good news for human brain health. The bad news is: CAT1 and CAT2 do play such a role. Therefore, they deserve to be mentioned in this context. We hope that our considerations will contribute to a more appropriate assessment of these transporters in further studies on healthy and diseased CNS.

\section{Conflicts of Interest}

No potential conflict of interest relevant to this article was reported.

\section{Author Contributions}

Conceptualization and writing: Hans-Gert Bernstein. Literature search and writing: Gerburg Keilhoff. Literature search: Henrik Dobrowolny. Supervision: Johann Steiner.

\section{ORCID}

Hans-Gert Bernstein https://orcid.org/0000-0002-2594-1689

Gerburg Keilhoff https://orcid.org/0000-0001-8836-2679

Henrik Dobrowolny https://orcid.org/0000-0003-3393-3037

Johann Steiner https://orcid.org/0000-0002-2611-2268

\section{REFERENCES}

1. Aykaç A, Şehirli AÖ. The role of the SLC transporters protein in the neurodegenerative disorders. Clin Psychopharmacol Neurosci 2020;18:174-187.

2. Fotiadis D, Kanai Y, Palacín M. The SLC3 and SLC7 families of amino acid transporters. Mol Aspects Med 2013;34:139-158.

3. Scalise M, Galluccio M, Console L, Pochini L, Indiveri C. The human SLC7A5 (LAT1): the intriguing histidine/large neutral amino acid transporter and its relevance to human health.
Front Chem 2018;6:243.

4. Morris ME, Rodriguez-Cruz V, Felmlee MA. SLC and ABC transporters: expression, localization, and species differences at the blood-brain and the blood-cerebrospinal fluid barriers. AAPS I 2017; 19:1317-1331.

5. Hu C, Tao L, Cao X, Chen L. The solute carrier transporters and the brain: physiological and pharmacological implications. Asian J Pharm Sci 2020;15:131-144.

6. Braissant O, Gotoh T, Loup M, Mori M, Bachmann C. Differential expression of the cationic amino acid transporter $2(B)$ in the adult rat brain. Brain Res Mol Brain Res 2001;91:189-195.

7. Jäger K, Wolf S, Dobrowolny H, Steiner J, Nave H, Maronde E, et al. Differential topochemistry of three cationic amino acid transporter proteins, hCAT1, hCAT2 and hCAT3, in the adult human brain. Amino Acids 2013;44:423-433.

8. Tachikawa M, Hirose S, Akanuma SI, Matsuyama R, Hosoya $\mathrm{KI}$. Developmental changes of I-arginine transport at the blood-brain barrier in rats. Microvasc Res 2018;17:16-21.

9. Bernstein HG, Jäger K, Dobrowolny H, Steiner J, Keilhoff G, Bogerts B, et al. Possible sources and functions of L-homoarginine in the brain: review of the literature and own findings. Amino Acids 2015;47:1729-1740.

10. Bernstein HG, Jäger K, Fiebig J, Wolf S, Wick M, Dobrowolny $\mathrm{H}$, et al. Agmatinase and human cationic amino acid transporter 1 in mood disorder: what's under the microscope? I Neurobehav Sci 2014;1:67-71.

11. Bernstein HG, Stich $C$, Jäger $K$, Dobrowolny $H$, Wick $M$, Steiner J, et al. Agmatinase, an inactivator of the putative endogenous antidepressant agmatine, is strongly upregulated in hippocampal interneurons of subjects with mood disorders. Neuropharmacology 2012;62:237-246.

12. Colton CA, Mott RT, Sharpe H, Xu Q, Van Nostrand WE, Vitek MP. Expression profiles for macrophage alternative activation genes in $A D$ and in mouse models of $A D$. J Neuroinflammation 2006;3:27. 